\title{
A!
}

This is an electronic reprint of the original article.

This reprint may differ from the original in pagination and typographic detail.

Rysti, J.; Mäkinen, J. T.; Autti, S.; Kamppinen, T.; Volovik, G. E.; Eltsov, V. B.

\section{Suppressing the Kibble-Zurek Mechanism by a Symmetry-Violating Bias}

Published in:

Physical Review Letters

DOI:

10.1103/PhysRevLett.127.115702

Published: 08/09/2021

Document Version

Publisher's PDF, also known as Version of record

Please cite the original version:

Rysti, J., Mäkinen, J. T., Autti, S., Kamppinen, T., Volovik, G. E., \& Eltsov, V. B. (2021). Suppressing the KibbleZurek Mechanism by a Symmetry-Violating Bias. Physical Review Letters, 127(11), [115702].

https://doi.org/10.1103/PhysRevLett.127.115702

This material is protected by copyright and other intellectual property rights, and duplication or sale of all or part of any of the repository collections is not permitted, except that material may be duplicated by you for your research use or educational purposes in electronic or print form. You must obtain permission for any other use. Electronic or print copies may not be offered, whether for sale or otherwise to anyone who is not an authorised user. 


\title{
Suppressing the Kibble-Zurek Mechanism by a Symmetry-Violating Bias
}

\author{
J. Rysti, ${ }^{1}$ J. T. Mäkinen $\odot,{ }^{1, *}$ S. Autti $\odot,{ }^{1,2}$ T. Kamppinen $\odot,{ }^{1}$ G. E. Volovik $\odot,{ }^{1,3}$ and V. B. Eltsov ${ }^{1}$ \\ ${ }^{1}$ Department of Applied Physics, Aalto University, POB 15100, FI-00076 AALTO, Espoo, Finland \\ ${ }^{2}$ Department of Physics, Lancaster University, Lancaster LA1 4YB, United Kingdom \\ ${ }^{3}$ L.D. Landau Institute for Theoretical Physics, Moscow, 142432, Russia
}

(Received 14 June 2021; accepted 16 July 2021; published 8 September 2021)

\begin{abstract}
The formation of topological defects in continuous phase transitions is driven by the Kibble-Zurek mechanism. Here we study the formation of single- and half-quantum vortices during transition to the polar phase of ${ }^{3} \mathrm{He}$ in the presence of a symmetry-breaking bias provided by the applied magnetic field. We find that vortex formation is suppressed exponentially when the length scale associated with the bias field becomes smaller than the Kibble-Zurek length. We thus demonstrate an experimentally feasible shortcut to adiabaticity - an important aspect for further understanding of phase transitions as well as for engineering applications such as quantum computers or simulators.
\end{abstract}

DOI: 10.1103/PhysRevLett.127.115702

In continuous phase transitions, random local choice of the symmetry-breaking order parameter leads to the formation of topological defects, such as quantized vortices. Originally a speculation in high-energy physics and cosmology [1], this mechanism, known as the Kibble-Zurek mechanism (KZM) [2-6], is now a cornerstone of out-ofequilibrium condensed matter physics. KZM has been observed in a range of systems such as superfluids, superconductors, and Bose condensates [7,8]. In the KZM scenario the transition takes place independently in various regions with the characteristic size depending on the transition rate. Each region inherits a random realization of the broken-symmetry feature of the new phase, such as the phase of the order parameter in a superfluid transition. When the expanding regions merge, topological defects, such as quantized vortices, are formed. The predicted power-law dependence of the defect density on the quench rate has been confirmed in superfluid helium $[9,10]$ as well as in other systems (see, e.g., [7,8,11]).

In the theory of broken-symmetry phase transitions, a symmetry-violating bias field plays an important role, initiating the choice between the different degenerate states [12]. Bias can in particular be applied to nonadiabatic thermodynamic [1,2] or quantum [5] phase transitions that result in the formation of topological defects via the KZM. It has been proposed that if the applied symmetry-breaking bias is sufficiently large, the adiabatic (defect-free) regime is restored [13]. The crossover from the Kibble-Zurek (KZ) regime to the adiabatic regime occurs at the characteristic value of the bias defined by the quench rate. Such crossover has been analyzed theoretically in a quantum phase transition in the Ising chain $[13,14]$ and in its classical counterpart [15]. Generally speaking, the KZM is expected to be modified in the presence of external factors such as inhomogeneities [27], or a propagating front of the phase transition [28]. Applying a bias allows for the external control of the magnitude of the KZM directly. Controlled restoration of the adiabatic regime by a symmetry-breaking bias can be utilized in applications requiring delicate and fast control of engineered quantum systems [13,29].

In this Letter we probe experimentally the use of an external bias for suppressing the formation of singlequantum vortices (SQV) and half-quantum vortices (HQV) [9,30,31] produced by the KZM in the phase transition from normal ${ }^{3} \mathrm{He}$ to the superfluid polar phase [32]. We report three central observations: (i) for HQVs the threshold bias for the onset of suppression is set by matching the characteristic length of the applied symmetrybreaking field to the Kibble-Zurek length, set by the quench rate; (ii) beyond the onset, the suppression takes over exponentially, with the onset threshold normalizing the bias field in the exponent; and (iii) the creation of SQVs is similarly suppressed for increasing bias fields while the threshold value is different from that for HQVs.

The spectrum of topological defects in the polar phase and the bias fields one can apply are understood in terms of the order parameter of the polar phase:

$$
A_{j \beta}=\Delta_{P} \hat{\mathbf{d}}_{j} \hat{\mathbf{m}}_{\beta} e^{i \Phi} .
$$

Here $\Delta_{P}$ is the maximum gap in the quasiparticle energy spectrum, and $\Phi$ is the superfluid phase. The unit vector $\hat{\mathbf{d}}$ determines the direction of the easy plane of the magnetic anisotropy and $\hat{\mathbf{m}}$ that of the orbital anisotropy. The anisotropy originates from $p$-wave Cooper pairing with the orbital momentum and spin of a pair equal to 1 . In the $p$-wave superfluid, confinement modifies the resulting order parameter [32-38]. The polar phase is stabilized within the confining nanomaterial, which consists of nearly parallel solid strands, and $\hat{\mathbf{m}}$ is pinned along the strand 


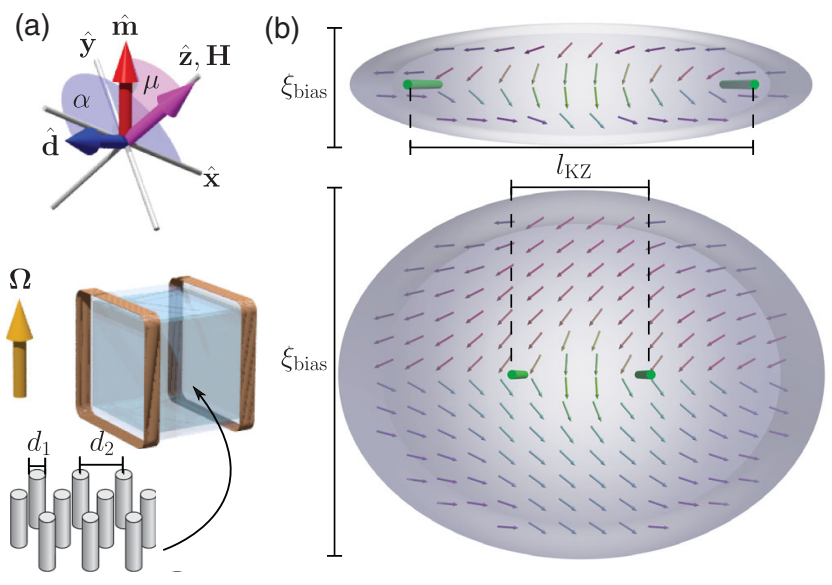

FIG. 1. Experimental principles. (a) The cubic $4 \times 4 \times 4 \mathrm{~mm}^{3}$ sample container is surrounded by NMR coils and filled with solid strands oriented along the vertical axis with the average diameter $d_{1}=9 \mathrm{~nm}$ [39] and average separation $d_{2} \approx 35 \mathrm{~nm}$. The space between the strands is filled with liquid ${ }^{3} \mathrm{He}$. The magnetic field $\mathbf{H} \| \hat{\mathbf{z}}$ can be applied in any direction in the plane transverse to the NMR coil axes (angle $\mu$ is represented by the light red sector). The orbital anisotropy vector $\hat{\mathbf{m}}$ is pinned along the confining strands, and the spin anisotropy vector $\hat{\mathbf{d}}$ is locked to the $\mathbf{x y}$ plane (light blue sector represents angle $\alpha$ ) by $\mathbf{H}$. The sample can be rotated about the vertical axis with the angular velocity $\boldsymbol{\Omega}$ up to $3 \mathrm{rad} \mathrm{s}^{-1}$. (b) The arrows represent the winding of $\hat{\mathbf{d}}$ by angle $\alpha$ (highlighted by the arrow colors) in the vicinity of two HQV cores (green cylinders). On a loop around an HQV core the $\hat{\mathbf{d}}$ vector rotates by $\pi$. For a large applied bias (top) pairs of HQVs are connected by narrow $\hat{\mathbf{d}}$ solitons (highlighted with the background color) and the width of the soliton, giving the characteristic length scale of the applied bias field $\xi_{\text {bias }}$, is much smaller than the $\mathrm{KZ}$ length, $\xi_{\text {bias }} \ll l_{\mathrm{KZ}}$, resulting in suppression of the HQV formation in the phase transition to the superfluid phase. For a vanishing bias (bottom) $\xi_{\text {bias }} \gg l_{\mathrm{KZ}}$, the winding of the $\hat{\mathbf{d}}$ vector is nearly uniform, and formation of HQVs in the phase transition is not suppressed.

direction, as shown in Fig. 1(a). The direction of $\hat{\mathbf{d}}$ is set by the competition between the magnetic anisotropy energy $\chi(\hat{\mathbf{d}} \cdot \mathbf{H})^{2} / 2$ in the magnetic field $\mathbf{H}$, and the spin-orbit interaction energy $g_{\mathrm{so}}(\hat{\mathbf{d}} \cdot \hat{\mathbf{m}})^{2}$, where $\chi$ is the magnetic susceptibility and $g_{\mathrm{so}}$ is the spin-orbit coupling.

In large magnetic fields $H^{2} \gg H_{\mathrm{so}}^{2}=g_{\mathrm{so}} \chi^{-1}$, the $\hat{\mathbf{d}}$ vector is kept in the plane perpendicular to $\mathbf{H}$, and the spin-orbit interaction takes the form

$$
F_{\text {so }}=g_{\text {so }} \sin ^{2} \mu \sin ^{2} \alpha,
$$

where $\mu$ is the angle between the magnetic field and the $\hat{\mathbf{m}}$ vector and $\alpha$ is the azimuthal angle of $\hat{\mathbf{d}}$ in the plane perpendicular to the magnetic field (see Fig. 1). For $\mu \neq 0$, the spin-orbit interaction in Eq. (2) lifts the degeneracy over $\alpha$, playing the role of the symmetry-violating bias.

We first study the effect of applying a symmetrybreaking bias via the spin-orbit coupling. For a magnetic field oriented along the strands $\mu=0$, the spin-orbit bias is absent and the symmetry-breaking scheme (ignoring $\mathrm{SO}(2)$ orbital rotations about $\hat{\mathbf{m}}$ ) is

$$
G=\mathrm{U}(1) \times \mathrm{SO}(2) \rightarrow \Upsilon=\mathrm{Z}_{2}
$$

Here $G$ describes the symmetries of normal ${ }^{3} \mathrm{He}, \mathrm{U}(1)$ is the symmetry under the phase transformation, and $\mathrm{SO}(2)$ is the symmetry under rotation in spin space about the axis of the magnetic field. $\Upsilon$ denotes the symmetry of the polar phase order parameter, where $Z_{2}$ is the spin rotation by $\pi$ (corresponding to the change $\hat{\mathbf{d}} \rightarrow-\hat{\mathbf{d}}$ ) accompanied by the phase change by $\pi$. Since the homotopy group $\pi_{1}(G / \Upsilon)=$ $Z \times Z \times Z_{2}$, this symmetry-breaking scheme leads to three types of topological defects: SQVs in the superfluid phase ( $\Phi$ field); spin vortices in the orientation of the spin anisotropy vector ( $\alpha$ field); and HQVs, where both the superfluid phase $\Phi$ and the angle of the spin anisotropy vector $\alpha$ change by $\pi$.

When the spin-orbit interaction is turned on $(\mu \neq 0)$, the $\mathrm{SO}(2)$ symmetry in Eq. (3) is explicitly violated, and one obtains the following symmetry-breaking scheme:

$$
\tilde{G}=\mathrm{U}(1) \rightarrow \tilde{\Upsilon}=1
$$

Now the homotopy group is $\pi_{1}(\tilde{G} / \tilde{\Upsilon})=Z$, which means that only SQVs remain stable, since they are not influenced by the spin-orbit interaction. Spin vortices and HQVs become termination lines of topological solitons [9,30,40-42], as illustrated in Fig. 1(b). Assuming only HQVs are present, $\hat{\mathbf{d}}$ solitons connect pairs of HQVs of the opposite $\hat{\mathbf{d}}$ winding.

The presence of the solitons can be detected and their total volume in the sample measured using the nuclear magnetic resonance (NMR) techniques. The bulk of the sample forms the main peak in the continuous-wave NMR spectrum at the frequency $\omega_{\text {main }}$ (see Fig. $S 1$ in Supplemental Material, Ref. [15]). The $\hat{\mathbf{d}}$ soliton provides a trapping potential for standing spin waves, seen as a satellite peak in the NMR spectrum at the frequency $\omega_{\text {sat }}$ [9]. The relative sizes of the main peak and the satellite are determined by the volume occupied by the $\hat{\mathbf{d}}$ solitons in the sample. We note that the vortices created by the KZM are randomly oriented, but in our case the vortex density is low and thus the soliton volume connecting two HQVs is simply defined by the intervortex distance [15]. Measuring the initial density of $\mathrm{KZ}$ defects has traditionally been difficult due to the fast annihilation of nonequilibrium defects at temperatures close to the phase transition $[3,4,10,43]$. In our experiments the confining strands pin vortices in place $[9,30,41]$, providing the observer a frozen window to the out-of-equilibrium physics of the phase transition and a direct measurement of the $\mathrm{KZ}$ vortex density. 
We calibrate the size of the satellite peak by preparing a state by a very slow cooldown through the critical temperature $T_{c}$ at $H=0$ while the sample is in rotation. This way we create HQVs with aerial density $n_{v}=4 \Omega \kappa^{-1}$, where $\Omega$ is the angular velocity, $\kappa=h /\left(2 m_{3}\right)$ is the quantum of circulation, $h$ is the Planck constant, and $m_{3}$ is the ${ }^{3} \mathrm{He}$ atom mass. The calibration gives the relative satellite size $I_{\text {sat }}=I_{0} \sqrt{\Omega}$, where $I_{0}=0.090 \mathrm{~s}^{1 / 2} \mathrm{rad}^{-1 / 2} \quad$ [15]. The intervortex distance assuming a square lattice is

$$
L=n_{v}^{-1 / 2}=\frac{1}{2} \sqrt{\kappa} \frac{I_{0}}{I_{\mathrm{sat}}} .
$$

We also use this relation to calculate the HQV density and intervortex distance for HQVs created purely by the KZM (i.e., for $\Omega=0$ ). The combined effect of rotation and KZM is discussed in the Supplemental Material, Ref. [15].

We control the spin-orbit bias by applying a fixed magnetic field of $H=11 \mathrm{mT}$ with transverse component $H_{\perp}=H \sin \mu$ during the cooldown through $T_{c}$. We repeat cooldowns for different $H_{\perp}$ and different cooldown rates, as shown in Fig. 2. We observe a constant satellite size for small $H_{\perp}$ and its gradual suppression for larger values of $H_{\perp}$. We suggest that the threshold field $H_{\perp t}$ where the suppression of the formation of HQVs starts is determined by comparing the Kibble-Zurek length $l_{\mathrm{KZ}}=a \xi_{0}\left(\tau_{Q} / \tau_{0}\right)^{1 / 4}$ with the characteristic length of the bias, $\xi_{\text {bias }}$, given by the thickness of the $\hat{\mathbf{d}}$ solitons. Here $a \sim 1$ fixes the exact length scale for the defect formation (in our measurements $a \approx 2.3$ [15]), the quench rate is $\tau_{Q}^{-1}=-d\left(T / T_{c}\right) /\left.d t\right|_{T=T_{c}}$, where $T$ is temperature, $t$ is time, $\xi_{0}$ is the superfluid coherence length at low temperature, $\tau_{0}=\xi_{0} v_{F}^{-1} \sim 1 \mathrm{~ns}$ is the order parameter relaxation time, $v_{F}$ is the Fermi velocity, $\xi_{\text {bias }} \sim \xi_{\text {so }} / \sin \mu$, and $\xi_{\text {so }}=17 \mu \mathrm{m}$ is the dipole length [9].

Equating $l_{\mathrm{KZ}}$ with $\xi_{\text {bias }}$ gives the following threshold bias for the suppression of HQV creation:

$$
H_{\perp t}=\frac{\xi_{\mathrm{so}}}{l_{\mathrm{KZ}}} H .
$$

In the spirit of Ref. [13] we propose that the defect density $\propto I_{\text {sat }}^{2}$ decays exponentially after the transition field. In terms of the satellite intensity, this reads as

$$
I_{\text {sat }}= \begin{cases}I_{\text {sat0 }} & \text { for } H_{\perp}<H_{\perp t} \\ I_{\text {sat } 0} \exp \left(1-H_{\perp} / H_{\perp t}\right) & \text { for } H_{\perp} \geq H_{\perp t},\end{cases}
$$

where $I_{\text {sat0 }}$ is the initial satellite intensity. We note that for this model $\int_{0}^{\infty} I_{\text {sat }} d H_{\perp}=2 I_{\text {sat } 0} H_{\perp t}$ and the numerical integral of the measured $I_{\text {sat }}$ can be used to determine $H_{\perp t}$ without fitting.

Our experiments, as shown in Fig. 2, confirm the validity of the model in Eq. (7). We use the zero-bias intervortex distance $\left.L\right|_{H_{\perp}=0}$, Eq. (5), as the measured value of $l_{\mathrm{KZ}}[9,43,44]$.

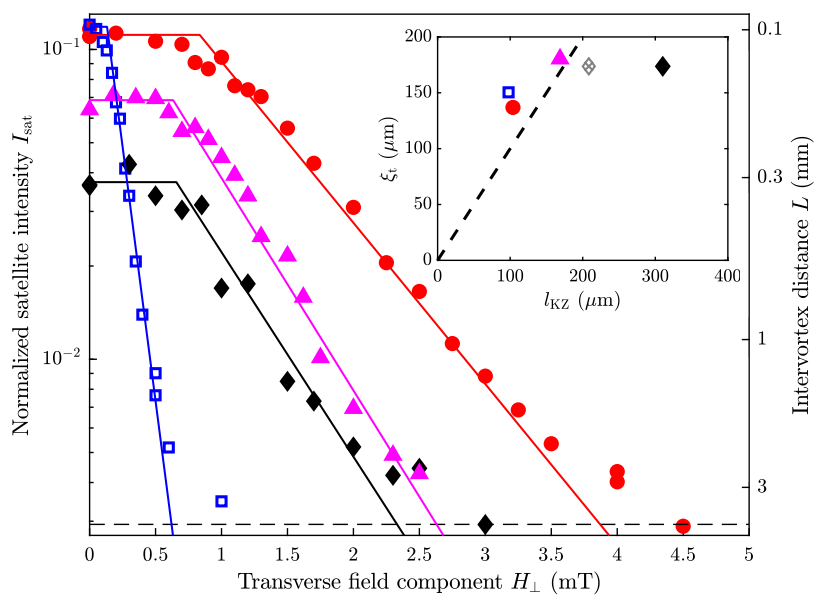

FIG. 2. Suppression of the HQV density created by the KZM as a function of the applied bias. Filled red circles, magenta triangles, and black diamonds correspond to quench rates of $\tau_{Q} \approx 3.8 \times 10^{2} \mathrm{~s}, \tau_{Q} \approx 1.4 \times 10^{3} \mathrm{~s}$, and $\tau_{Q} \approx 7.7 \times 10^{3} \mathrm{~s}$, respectively, while applying a constant $H=11 \mathrm{mT}$ magnetic field. The field is rotated to achieve different bias fields $H_{\perp}=H \sin \mu$. Open blue squares $\left(\tau_{Q} \approx 6.0 \times 10^{2} \mathrm{~s}\right)$ correspond to measurements with zero axial field component, $H_{\perp}=H$. Vortex density is constant for $H_{\perp}<H_{\perp t}$ and suppressed for higher bias fields. The suppression starts when the characteristic length scale of the bias field $\xi_{\text {bias }}\left(H_{\perp}\right)$ becomes smaller than the relevant KibbleZurek length. Solid lines correspond to theoretical model (see text for details). The dashed line shows where the intervotex distance becomes comparable with the container size. The inset shows the extracted threshold bias length $\xi_{t}$ as a function of $l_{\mathrm{KZ}}$ with the same symbols. The dashed line is $\xi_{t}=l_{\mathrm{KZ}}$. The patterned gray diamond is the same measurement as the black diamond, but with $l_{\mathrm{KZ}}$ on the horizontal axis replaced with an estimation of the transition front thickness $l_{F}[15]$. For other measurements, $l_{F}$ lies beyond the right border of the plot.

We emphasize that the threshold field $H_{\perp t}$, which also normalizes the exponent, is determined by integration of the experimental data without a fitting procedure. The result agrees well with the conjecture $\xi_{t} \equiv \xi_{\text {bias }}\left(H_{\perp t}\right)=l_{\mathrm{KZ}}$.

The result for the slowest quench rate deviates, however, from this dependence. In the presence of a thermal gradient, the phase transition proceeds via a propagating front, where the ordering of the low-temperature phase lags behind the temperature front where $T=T_{c}$ by distance $l_{F}$. The KZM operates in the band of width $l_{F}$ and is modified in comparison to the homogeneous cooling scenario $[28,45,46]$. As $\tau_{Q}$ increases, $l_{F}$ decreases and $l_{\mathrm{KZ}}$ increases. We suggest that the smaller of the two characteristic lengths $l_{\mathrm{KZ}}$ and $l_{F}$ determines the threshold bias $\xi_{t}$. We estimate that in our measurement $l_{F}<l_{\mathrm{KZ}}$ only for the slowest quench rate (black diamonds in Fig. 2) for which $l_{F} \sim 210 \mu \mathrm{m}$ (gray patterned diamond in Fig. 2, inset), matching the observed value of $\xi_{t}$ [15].

Alternatively we can apply a direct field bias with a weak magnetic field oriented perpendicular to $\hat{\mathbf{m}}$. The small magnetic field $H_{\perp}<H_{\text {so }}$ violates the symmetry under 
rotation about $\hat{\mathbf{m}}$, which leads to the formation of solitons, absent at zero magnetic field, with the soliton thickness now determined by the magnetic field directly, $\xi_{\text {bias }}=\xi_{H}=\xi_{\text {so }} H_{\text {so }} / H$. Equating $\xi_{\text {bias }}$ with $l_{\mathrm{KZ}}$ yields a criterion for the threshold field similar to that in Eq. (6) but with $H$ replaced by $H_{\mathrm{so}}$. The expected decrease of the threshold field in this case is $H_{\mathrm{so}} / H=\Omega_{P} / \omega_{\text {main }} \approx$ $\sqrt{2\left(1-\omega_{\text {sat }} / \omega_{\text {main }}\right)} \approx 0.17$ [9], where $\Omega_{P}$ is the polar phase Leggett frequency. It is confirmed experimentally by the blue squares in Fig. 2. Here the ratio of the threshold field relative to the red circles, which correspond to the spin-orbit bias with similar quench rate, is 0.16 .

Finally, we study the fate of SQVs under the symmetrybreaking bias created by tilting the magnetic field. Because of the absence of the topological solitons one would naïvely expect that the bias has no effect on the KZM for SQVs. Without the solitons, SQVs are not seen in continuouswave NMR, but they were found to increase the relaxation rate of a magnon Bose-Einstein condensate (BEC) [41,47]. Independent measurements with $\mathrm{SQV}$ s created by rotation indicate that the BEC relaxation rate increases monotonically when the SQV density grows [41]. In our measurements we create both HQVs and SQVs by the KZM and subtract the effect of HQVs by using a calibration of the relaxation rate of the magnon BEC with respect to the satellite intensity [see Fig. 3(b) from Ref. [41]]. The remaining contribution to the relaxation we attribute to SQVs. This contribution shows the characteristic dependence with a threshold and exponential suppression akin to Eq. (7), as shown in Fig. 3. A possible explanation for this behavior is that an applied bias influences the structure of the vortex core. For example in the superfluid B phase, the $\mathrm{SO}(2)$ symmetry of the SQV core is spontaneously broken at low temperatures, and the core transforms into a pair of tightly bound half-quantum cores [48-50]. In the polar phase, the spin-orbit interaction and magnetic anisotropy may play the role of the symmetry-violating bias for the phase transitions inside the vortex core, but the detailed investigation remains a task for the future.

In conclusion, we report a crossover from the KibbleZurek regime of HQV creation to the adiabatic regime, where vortex formation is rapidly suppressed by a symmetry-violating bias. We thus demonstrate an experimentally feasible shortcut to adiabaticity, where the adiabatic regime can be reached without an infinitely slow transition rate. In our experiments the symmetry-violating bias is provided either by the spin-orbit interaction or directly by external magnetic field. The crossover to the adiabatic regime takes place when the characteristic length scale of the bias, given by the thickness of the topological solitons connecting neighboring HQVs, becomes smaller than the Kibble-Zurek length determined by the transition rate or the thickness of the transition front in the case of slow inhomogeneous cooling. Beyond the onset, the suppression of the KZM takes place exponentially. We also

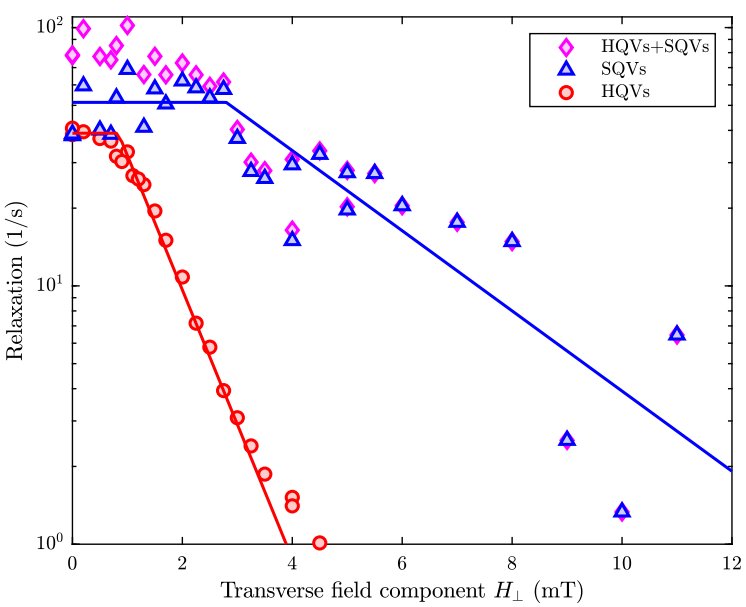

FIG. 3. Suppression of SQV density as a function of the applied bias. The measured magnon BEC relaxation rate (magenta diamonds) includes contributions from HQVs and SQVs. The HQV contribution (red circles) is separated using linear NMR measurements of the satellite intensity and calibration from Ref. [41]. The remaining contribution to relaxation (blue triangles) we attribute to SQVs. The observed relaxation rates are compared with the suppression model [solid lines, Eq. (7)], and the red line corresponds to the same threshold as in Fig. 2. In these measurements the quench rate is $\tau_{Q} \sim 4 \times 10^{2} \mathrm{~s}$, and the magnitude of the magnetic field is kept constant while its direction is varied. The largest transverse field value corresponds to $\mu=\pi / 2$. Constant BEC relaxation rate not related to vortices has been subtracted.

report similar suppression of SQV formation by the $\mathrm{KZM}$, indicating that there may be a symmetry-breaking transition in the SQV core, sensitive to the symmetryviolating external bias.

The symmetry-breaking aspect of the bias field is essential for the suppression of the KZM, which otherwise is very robust. As an example [15], we show that adding an array of HQVs created by rotating the sample has no effect on the KZM even when the characteristic length scale of the added lattice becomes smaller than the Kibble-Zurek length. We also note that HQVs are composite defects, whose KZM formation is rarely studied experimentally, and that they are analogs of Alice strings [51-53]. The KZM formation of HQVs studied here may shed light on defect formation across phase transitions in theories considering such systems.

Our results can be generalized to the bias-induced restoration of adiabadicity in various phase transitions including quantum phase transitions, which could provide applications for technologies such as quantum simulators and computers $[13,29]$. On a more speculative note, it is not excluded that the bias plays a role in the so-called collapse of the wave function in quantum mechanics. In principle, the latter can be seen as "phase transition" occurring in the continuous spectrum of an infinite system [54-56]. One of the many quantum states participating in a given quantum 
superposition is perhaps then selected by the infinitesimal bias unavoidably present in any experiment.

We thank V. V. Dmitriev for providing the NAFEN sample. This Letter has been supported by the European Research Council (ERC) under the European Union's Horizon 2020 research and innovation program (Grant Agreement No. 694248) and by Academy of Finland Project No. 332964. S. A. acknowledges support from the Jenny and Antti Wihuri Foundation via the Council of Finnish Foundations, and T. K. acknowledges support from the Finnish Cultural Foundation. This research made use of the OtaNano Low Temperature Laboratory infrastructure of Aalto University, which is part of the European Microkelvin Platform (European Union's Horizon 2020 Grant No. 824109).

\section{J.R. and J. T. M. contributed equally to this work}

Note added in the proof.-A recent experimental investigation [57] studies the saturated regime of defect density in a rapidly quenched Bose gas, suggesting that in the saturated regime the initial defect density is determined by the early time coarsening dynamics of the emerging order parameter. The study of the combined effect of the saturation of defect density and an applied bias remains an interesting topic for the future.

*jere.makinen@aalto.fi

[1] T. W. B. Kibble, Topology of cosmic domains and strings, J. Phys. A 9, 1387 (1976).

[2] W.H. Zurek, Cosmological experiments in superfluid helium? Nature (London) 317, 505 (1985).

[3] V. M. H. Ruutu, V. B. Eltsov, A. J. Gill, W. B. Kibble, M. Krusius, Y. G. Makhlin, B. Placais, G. E. Volovik, and W. $\mathrm{Xu}$, Vortex formation in neutron-irradiated superfluid ${ }^{3} \mathrm{He}$ as an analogue of cosmological defect formation, Nature (London) 382, 334 (1996).

[4] C. Bauerle, Y. M. Bunkov, S. N. Fisher, H. Godfrin, and G. R. Pickett, Laboratory simulation of cosmic string formation in the early Universe using superfluid ${ }^{3} \mathrm{He}$, Nature (London) 382, 332 (1996).

[5] J. Dziarmaga, Dynamics of a Quantum Phase Transition: Exact Solution of the Quantum Ising Model, Phys. Rev. Lett. 95, 245701 (2005).

[6] M. Prüfer, P. Kunkel, H. Strobel, S. Lannig, D. Linnemann, C.-M. Schmied, J. Berges, T. Gasenzer, and M. Oberthaler, Observation of universal dynamics in a spinor Bose gas far from equilibrium, Nature (London) 563, 217 (2018).

[7] A. Polkovnikov, K. Sengupta, A. Silva, and M. Vengalattore, Colloquium: Nonequilibrium dynamics of closed interacting quantum systems, Rev. Mod. Phys. 83, 863 (2011).

[8] A. Del Campo and W. Zurek, Universality of phase transition dynamics: Topological defects from symmetry breaking, Int. J. Mod. Phys. A 29, 1430018 (2014).

[9] S. Autti, V. V. Dmitriev, J. T. Mäkinen, A. A. Soldatov, G. E. Volovik, A. N. Yudin, V. V. Zavjalov, and V. B. Eltsov,
Observation of Half-Quantum Vortices in Topological Superfluid ${ }^{3} \mathrm{He}$, Phys. Rev. Lett. 117, 255301 (2016).

[10] Y. M. Bunkov, A. I. Golov, V. S. L'vov, A. Pomyalov, and I. Procaccia, Evolution of a neutron-initiated micro big bang in superfluid ${ }^{3} \mathrm{He}-B$, Phys. Rev. B 90, 024508 (2014).

[11] J. Beugnon and N. Navon, Exploring the Kibble-Zurek mechanism with homogeneous Bose gases, J. Phys. B 50, 022002 (2017).

[12] Y. G. Sinai, Theory of Phase Transitions. Rigorous Results (Pergamon Press, New York, 1982).

[13] M. M. Rams, J. Dziarmaga, and W. H. Zurek, Symmetry Breaking Bias and the Dynamics of a Quantum Phase Transition, Phys. Rev. Lett. 123, 130603 (2019).

[14] B. Yan, V. Y. Chernyak, W. H. Zurek, and N. A. Sinitsyn, Nonadiabatic Phase Transition with Broken Chiral Symmetry, Phys. Rev. Lett. 126, 070602 (2021).

[15] See Supplemental Material at http://link.aps.org/ supplemental/10.1103/PhysRevLett.127.115702 for details, which includes Refs. [16-26].

[16] P. J. Heikkinen, S. Autti, V. B. Eltsov, R. P. Haley, and V. V. Zavjalov, Microkelvin thermometry with Bose-Einstein condensates of magnons and applications to studies of the $\mathrm{AB}$ interface in superfluid ${ }^{3} \mathrm{He}$, J. Low Temp. Phys. 175, 681 (2014).

[17] E. V. Thuneberg, Hydrostatic theory of superfluid ${ }^{3} \mathrm{He}-\mathrm{B}$, J. Low Temp. Phys. 122, 657 (2001).

[18] P. J. Hakonen, M. Krusius, M. M. Salomaa, R. H. Salmelin, J. T. Simola, A. D. Gongadze, G. E. Vanchnadze, and G. A. Kharadze, NMR and axial magnetic field textures in stationary and rotating superfluid ${ }^{3} \mathrm{He}-\mathrm{B}$, J. Low Temp. Phys. 76, 225 (1989).

[19] R. Blaauwgeers, M. Blažková, M. Človečko, V. B. Eltsov, R. de Graaf, J. Hosio, M. Krusius, D. Schmoranzer, W. Schoepe, L. Skrbek, P. Skyba, R. E. Solntsev, and D. E. Zmeev, Quartz tuning fork: Thermometer, pressureand viscometer for helium liquids, J. Low Temp. Phys. 146, 537 (2007).

[20] M. Blažková, M. Človečko, V. B. Eltsov, E. Gažo, R. de Graaf, J. J. Hosio, M. Krusius, D. Schmoranzer, W. Schoepe, L. Skrbek, S. P. R. E. Solntsev, and W. F. Vinen, Vibrating quartz fork-A tool for cryogenic helium research, J. Low Temp. Phys. 150, 525 (2008).

[21] T. S. Riekki, J. Rysti, J. T. Mäkinen, A. P. Sebedash, V. B. Eltsov, and J. T. Tuoriniemi, Effects of ${ }^{4} \mathrm{He}$ film on quartz tuning forks in ${ }^{3} \mathrm{He}$ at ultra-low temperatures, J. Low Temp. Phys. 196, 73 (2019).

[22] V. V. Dmitriev, A. A. Soldatov, and A. N. Yudin, Effect of Magnetic Boundary Conditions on Superfluid ${ }^{3} \mathrm{He}$ in Nematic Aerogel, Phys. Rev. Lett. 120, 075301 (2018).

[23] I. S. Aranson, N. B. Kopnin, and V. M. Vinokur, Dynamics of vortex nucleation by rapid thermal quench, Phys. Rev. B 63, 184501 (2001).

[24] D. S. Greywall, Thermal conductivity of normal liquid ${ }^{3} \mathrm{He}$, Phys. Rev. B 29, 4933 (1984).

[25] R. Dobbs, Helium Three (Oxford University Press, New York, 2000).

[26] V. V. Dmitriev, L. A. Melnikovsky, A. A. Senin, A. A. Soldatov, and A. N. Yudin, Anisotropic spin diffusion in liquid ${ }^{3} \mathrm{He}$ confined in nafen, JETP Lett. 101, 808 (2015). 
[27] A. Del Campo, T. Kibble, and W. Zurek, Causality and nonequilibrium second-order phase transitions in inhomogeneous systems, J. Phys. Condens. Matter 25, 404210 (2013).

[28] T. W. B. Kibble and G. E. Volovik, On phase ordering behind the propagating front of a second-order transition, JETP Lett. 65, 102 (1997).

[29] A. del Campo and K. Kim, Focus on shortcuts to adiabaticity, New J. Phys. 21, 050201 (2019).

[30] J. T. Mäkinen, V. V. Dmitriev, J. Nissinen, J. Rysti, G. E. Volovik, A. N. Yudin, K. Zhang, and V. B. Eltsov, Halfquantum vortices and walls bounded by strings in the polar-distorted phases of topological superfluid ${ }^{3} \mathrm{He}$, Nat. Commun. 10, 237 (2019).

[31] R. C. Regan, J. J. Wiman, and J. A. Sauls, Half-quantum vortices in nematic and chiral phases of ${ }^{3} \mathrm{He}$, arXiv:2105 .01257.

[32] V. V. Dmitriev, A. A. Senin, A. A. Soldatov, and A. N. Yudin, Polar Phase of Superfluid ${ }^{3} \mathrm{He}$ in Anisotropic Aerogel, Phys. Rev. Lett. 115, 165304 (2015).

[33] L. V. Levitin, R. G. Bennett, A. Casey, B. Cowan, J. Saunders, D. Drung, T. Schurig, and J. M. Parpia, Phase diagram of the topological superfluid ${ }^{3} \mathrm{He}$ confined in a nanoscale slab geometry, Science 340, 841 (2013).

[34] J. I. A. Li, A. M. Zimmerman, J. Pollanen, C. A. Collett, and W. P. Halperin, Anisotropic Phases of Superfluid ${ }^{3} \mathrm{He}$ in Compressed Aerogel, Phys. Rev. Lett. 114, 105302 (2015).

[35] J. I. A. Li, J. Pollanen, A. M. Zimmerman, C. A. Collett, W. J. Gannon, and W. P. Halperin, The superfluid glass phase of ${ }^{3}$ He-A, Nat. Phys. 9, 775 (2013).

[36] L. V. Levitin, B. Yager, L. Sumner, B. Cowan, A. J. Casey, J. Saunders, N. Zhelev, R. G. Bennett, and J. M. Parpia, Evidence for a Spatially Modulated Superfluid Phase of ${ }^{3} \mathrm{He}$ under Confinement, Phys. Rev. Lett. 122, 085301 (2019).

[37] A. J. Shook, V. Vadakkumbatt, P. Senarath Yapa, C. Doolin, R. Boyack, P. H. Kim, G. G. Popowich, F. Souris, H. Christani, J. Maciejko, and J. P. Davis, Stabilized Pair Density Wave via Nanoscale Confinement of Superfluid ${ }^{3}$ He, Phys. Rev. Lett. 124, 015301 (2020).

[38] V. V. Dmitriev, M. S. Kutuzov, A. A. Soldatov, and A. N. Yudin, Superfluid $\beta$ phase in liquid ${ }^{3} \mathrm{He}$, arXiv:2012.06302.

[39] V. E. Asadchikov, R. S. Askhadullin, V. V. Volkov, V. V. Dmitriev, N. K. Kitaeva, P. N. Martynov, A. A. Osipov, A. A. Senin, A. A. Soldatov, D. I. Chekrygina, and A. N. Yudin, Structure and properties of "nematically ordered" aerogels, JETP Lett. 101, 556 (2015).

[40] V. P. Mineyev and G. E. Volovik, Planar and linear solitons in superfluid ${ }^{3} \mathrm{He}$, Phys. Rev. B 18, 3197 (1978).

[41] S. Autti, J. T. Mäkinen, J. Rysti, G. E. Volovik, V. V. Zavjalov, and V.B. Eltsov, Exceeding the Landau speed limit with topological Bogoliubov Fermi surfaces, Phys. Rev. Research 2, 033013 (2020).

[42] G. E. Volovik, J. Rysti, J. T. Mäkinen, and V. B. Eltsov, Spin, orbital, Weyl and other glasses in topological superfluids, J. Low Temp. Phys. 196, 82 (2019).

[43] V. B. Eltsov, M. Krusius, and G. E. Volovik, Vortex formation and dynamics in superfluid ${ }^{3} \mathrm{He}$ and analogies in quantum field theory, in Progress in Low Temperature Physics (Elsevier, New York, 2005), Vol. XV, pp. 1-137, https://doi.org/10.1016/S0079-6417(05)15001-X.

[44] C. Bäuerle, Y. M. Bunkov, S. N. Fisher, H. Godfrin, and G. R. Pickett, Superfluid ${ }^{3} \mathrm{He}$ simulation of cosmic string creation in the early Universe, J. Low Temp. Phys. 110, 13 (1998).

[45] N. B. Kopnin and E. V. Thuneberg, Time-Dependent Ginzburg-Landau Analysis of Inhomogeneous Normal-Superfluid Transitions, Phys. Rev. Lett. 83, 116 (1999).

[46] C.-R. Yi, S. Liu, R.-H. Jiao, J.-Y. Zhang, Y.-S. Zhang, and S. Chen, Exploring Inhomogeneous Kibble-Zurek Mechanism in a Spin-Orbit Coupled Bose-Einstein Condensate, Phys. Rev. Lett. 125, 260603 (2020).

[47] S. Autti, V. V. Dmitriev, J. T. Mäkinen, J. Rysti, A. A. Soldatov, G. E. Volovik, A. N. Yudin, and V. B. Eltsov, Bose-Einstein Condensation of Magnons and Spin Superfluidity in the Polar Phase of ${ }^{3} \mathrm{He}$, Phys. Rev. Lett. 121, 025303 (2018).

[48] E. V. Thuneberg, Identification of vortices in superfluid ${ }^{3}$ He- $B$, Phys. Rev. Lett. 56, 359 (1986).

[49] M. M. Salomaa and G. E. Volovik, Vortices with spontaneously broken axisymmetry in ${ }^{3} \mathrm{He}-B$, Phys. Rev. Lett. 56, 363 (1986).

[50] M. M. Salomaa and G. E. Volovik, Quantized vortices in superfluid ${ }^{3} \mathrm{He}$, Rev. Mod. Phys. 59, 533 (1987).

[51] Y. Fujimoto and M. Nitta, Non-Abelian Alice strings in two-flavor dense QCD, Phys. Rev. D 103, 054002 (2021).

[52] C. Chatterjee and M. Nitta, BPS Alice strings, J. High Energy Phys. 09 (2017) 046.

[53] T. Okada and Y. Sakatani, Defect branes as Alice strings, J. High Energy Phys. 03 (2015) 131.

[54] M. Grady, Spontaneous symmetry breaking as the mechanism of quantum measurement, arXiv:hep-th/9409049.

[55] G. Volovik, Quantum analogues: From phase transitions to black holes and cosmology, Lect. Notes Phys. 718, 31 (2007).

[56] H. Ziaeepour, Quantum mechanics in symmetry language, arXiv:1305.4349.

[57] J. Goo, Y. Lim, and Y. Shin, preceding Letter, Defect Saturation in a Rapidly Quenched Bose Gas, Phys. Rev. Lett. 127, 115701 (2021). 